\title{
Efficient facial emotion recognition model using deep convolutional neural network and modified joint trilateral filter
}

\author{
Naveen Kumari ${ }^{1, *}$ and Rekha Bhatia ${ }^{1}$ \\ ${ }^{1}$ Department of computer science, Punjabi University regional centre for IT and Management, Mohali, India. \\ ${ }^{*}$ Corresponding author's email: naveencse2k4@gmail.com
}

\begin{abstract}
Facial emotion recognition extracts the human emotions from the images and videos. As such, it requires an algorithm to understand and model the relationships between faces and facial expressions, and to recognize human emotions. Recently, deep learning models are extensively utilized enhance the facial emotion recognition rate. However, the deep learning models suffer from the overfitting issue. Moreover, deep learning models perform poorly for images which have poor visibility and noise. Therefore, in this paper, a novel deep learning based facial emotion recognition tool is proposed. Initially, a joint trilateral filter is applied to the obtained dataset to remove the noise. Thereafter, contrast-limited adaptive histogram equalization (CLAHE) is applied to the filtered images to improve the visibility of images. Finally, a deep convolutional neural network is trained. Nadam optimizer is also utilized to optimize the cost function of deep convolutional neural networks. Experiments are achieved by using the benchmark dataset and competitive human emotion recognition models. Comparative analysis demonstrates that the proposed facial emotion recognition model performs considerably better compared to the competitive models.

Keywords: Image, Emotions, Neural networks, Labeling, Deep learning
\end{abstract}

\section{Introduction}

Extracting visual information from images is one of the fundamental skills of human intelligence [1]. With the advancement in artificial intelligence, it is assumed that robots can understand the feelings of human beings. However, recognizing emotions in an efficient manner is defined as ill-posed problem [2]. Human emotions can be recognized by considering various methods such as face recognition, speech recognition, physiological signals, etc. Speech and facial expressions are the natural methods to recognize emotions [3]. Many emotion recognition techniques are designed and implemented in the literature to recognize human emotions [4]. In this paper, our main concentration is to recognize the facial emotions.

The objective is to recreate a similar level of intellectual ability in artificial intelligence, which has motivated researchers from computer vision and natural language communities to design automatic emotion 
recognition systems [5]. Facial emotion recognition represents the content of an input image in the form of human emotions by using various machines and deep learning models [6]. Thus, it initially extracts the face information and thereafter, it provides a descriptive emotion [7]. Recently, many Convolutional Neural Networks (CNN) and Recurrent Neural Network (RNN) based emotion recognition models have been designed and implemented [8].

Li and Deng [9] proposed Artificial neural network (ANN) model to recognize facial emotions. Zhang et al. [10] designed spatial-temporal recurrent neural network to recognize facial emotions. Kim et al. [11] used hierarchical deep learning (HDL) to extract adaptive facial features to obtain better results.

From the existing literature, it has been found that the Decision tree (DT) [12, 13], Support Vector Machine (SVM) [14, 15], Random Forest (RF) [16, 17], and Artificial Neural Network (ANN) [9] are commonly utilized to recognize the human emotions. Jain et al. (2019) [18] designed deep neural networks (DNNs) using deep residual blocks. Wang et al. (2020) [19] combined CNN and RNN (CCNNRNN) to classify the human emotions. Arpita et al. (2020) [20] implemented a ResNet and attention block (CRAB) based human emotion recognition model. Lakshmi et al. (2021) [21] implemented a modified histogram of oriented gradients (HOG) and local binary pattern (LBP), i.e., HOGLBP to extract the features. Although these methods achieve good performance, but suffer from the overfitting issue. Moreover, these models perform poorly for images which have poor visibility and noise.

The main contributions of this paper are as follows:

1. A novel deep learning based facial emotion recognition tool is proposed.

2. The joint trilateral filter is applied on the obtained dataset to remove the noise.

3. Contrast limited adaptive histogram equalization (CLAHE) is applied to the filtered images to improve the visibility of images.

4. Nadam optimizer is also utilized to optimize the cost function of deep convolutional neural networks.

5. Experiments are considered by using the benchmark dataset and competitive human emotion recognition models.

The remainder of the paper is structured as follows: Section 2 discusses the related work. Section 3 mathematically defines the proposed model. Comparative results are discussed in Section 4. Concluding remarks are presented in Section 5.

\section{Related work}

Hung et al. [22] used multilevel transfer learning based on a fine-tune approach to recognize facial emotion. Lakshmi et al. [23] recognized facial emotion by utilizing Whale optimization algorithm (WOA) and teaching-learning-based optimization (TLBO). In this, Multi-Support Vector Neural Network (MultiSVNN) 
was used to build the model based on WOA and TLBO. Liu et al. [24] captured human facial emotions using multi-channel electroencephalography (EEG) signals and textual feature fusion method. Using both frequency and spatial domains, features were extracted. The model was trained using the SVM to recognize the facial emotions. Ngai et al. [25] enhanced facial recognition using 2-channel EEG and eye modality. CNN model was used to recognize and classify facial emotions.

Zhang et al. [26] proposed a model to recognize facial emotions using the correlation emotion label distribution learning. The six basic facial expressions were learned using a constructed convolutional neural network. Chen et al. [27] recognized the facial emotions by applying a softmax regression model. In this, facial expressions were learned through a deep sparse autoencoder network. It overcomes the issues such as gradient diffusion and local extrema in training of a model.Tan et al. [28] recognized facial expression using EEG and multimodal emotion recognition method. The issue of small dataset was resolved using Monte Carlo strategy that further improved the results. In this, recognition rate was achieved near to $83.33 \%$.

Wang et al. [19] fused speech features and facial expressions using bimodal fusion method to recognize human emotion. The facial emotions were recognized by combining RNN and CNN. The speech emotions were captured using CNN and LSTM. After that, facial and speech emotions were fused using a weighted decision fusion method. Li et al. [29] applied ResNet-50 to capture the facial emotions of humans. This helped to improve the robustness and generalization ability of the recognition models. Lakshmi et al. [21] proposed hybrid model to recognize facial emotions. In this, face regions were selected through ViolaJones method. Then, the required features are extracted from selected regions using local binary pattern and histogram of oriented gradients. Deep stack auto encoder was utilized to reduce the dimensionality of extracted features. Lastly, Multi-class SVM was applied to recognize and classify the emotions.

Deng et al. [30] recognized the facial emotions using Conditional generative adversarial network-based approach (cGAN). Zhang 9187342 used a multimodal face recognition model with EEG and deep automatic encoder. The objective features were selected using a decision tree. Du et al. [31] detected the emotions of players using facial expression and heart beat signals. Heart rate signals were learned through Bidirectional long and short term memory (Bi-LSTM) networks. Facial features were learned via CNN. Li et al. [32] recognized the continuous emotions by combining the facial expression and EEG. Choi et al. [33] used metric learning to recognize the continuous emotions. Hua et al. [34] used ensembled deep learning model to recognize facial expressions. Choi et al. [35] utilized 2-D landmark feature map to recognize facial micro-expression.

From the related work, it has been observed that the majority of the existing methods achieve good performance, but suffer from the overfitting issue. Additionally, the existing models perform poorly for images which have poor visibility and noise. 


\section{Proposed methodology}

In this paper, a novel deep convolutional neural network model is proposed to recognize the human emotions from facial images. The proposed model can dynamically focus on the salient features in the images during the training process. Initially, the joint trilateral filter is applied to the obtained dataset to remove the noise. Thereafter, CLAHE is applied to the filtered images to improve the visibility of images. Finally, a deep convolutional neural network is used for training the emotion recognition model. Nadam optimizer is also utilized to optimize the cost function of deep convolutional neural networks.

\subsection{Modified joint trilateral filter}

To remove the noise from images, a modified joint trilateral filter is proposed. It is an edge preserving filter which does not introduce various types of artifacts on the filtered images. Initially, a guided image $G_{d}$ i.e., the actual image $I_{\kappa}$ itself is considered. Assume that $I_{v a l}$ and $G_{v a l}$ are the illumination values at pixel $q$ and the guided image. $W_{r}$ is a kernel window at $k$ dependent on the bilateral filter [36, 37]. The modified joint trilateral filter can be defined as:

$$
J_{t f}\left(I_{\kappa}\right) \quad=\quad \frac{1}{\sum_{q \in K_{r}} M_{\theta}^{p q}\left(G_{d}\right)}\left(\sum_{q \in k_{r}} M_{\theta}^{p q}\left(G_{d}\right) \quad \times \quad I_{q} \times \sigma^{2}\left(I_{q}, G_{d}\right)\right)
$$

Here, the kernel weight function $\left(M_{\theta}^{p q}\left(G_{d}\right)\right)$ can be evaluated as:

$$
M_{\theta}^{p q}\left(G_{d}\right)=\frac{1}{|n|^{2}} \sum_{n:(p, q) \in k_{r}}\left(1+\frac{\left(G_{d p}-\mu_{n}\right)\left(G_{d q}-\mu_{n}\right)}{\sigma_{n}^{2}+\epsilon}\right)
$$

Here, $\mu_{n}$ and $\sigma_{n}^{2}$ show the mean and variance of $G_{d}$ in the local window $k_{r} .|n|$ defines the total number of pixels in window. When $G_{d_{p}}$ and $G_{d_{q}}$ are on the identical sides of an edge, the weight assigned to pixel $q$ is maximum. When $G_{d p}$ and $G_{d q}$ are on diverse sides, a minimum weight can be assigned to the pixel $q$.

\subsection{Contrast limited adaptive histogram equalization}

Reducing the effect of numerous lighting circumstances is a nontrivial issue in the field of image processing. The precision of emotion recognition is generally low if the visibility of the face changes. However, the standard histogram equalization based approaches are not effective for uneven lightening conditions as they may result in overenhanced images [38]. CLAHE has the ability to overcome the excessive amplification of noise by limiting the contrast. It can be implemented as follows:

$$
\begin{aligned}
f(D)= & (1-\omega y)\left((1-\omega x) f_{u l}(D)+\omega x f_{b l}(D)\right) \\
& +\omega y\left((1-\omega x) f_{u r}(D)+\omega x f_{b r}(D)\right)
\end{aligned}
$$


For every pixel, the mapping of four adjacent values of the histogram cumulative distribution function (CDF) to the pixel are desirable. $\omega x$ and $\omega y$ show the distance between pixels and the center of the left upper mask.

\subsection{Deep convolutional neural network}

Recently, CNN models have been extensively utilized to classify the computer vision problems [39, 40]. Various filters are utilized to compute potential features. Therefore, it achieves better performances than layers.

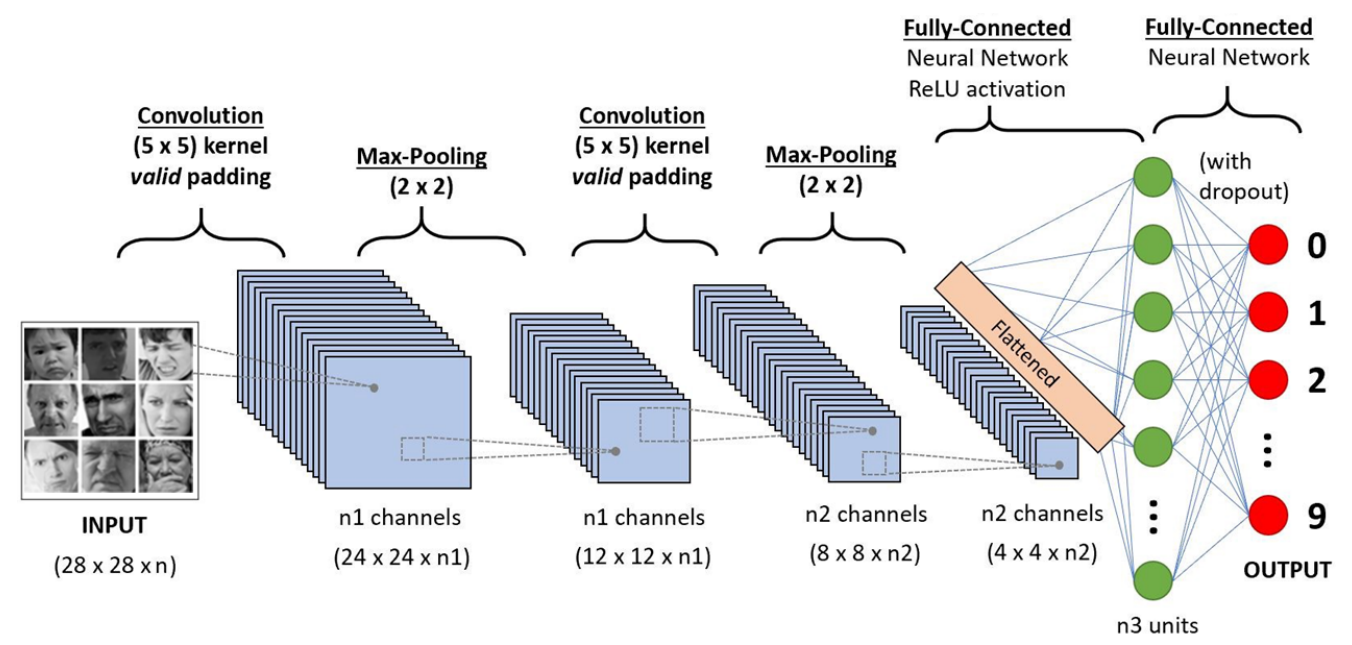

Figure 1: Diagrammatic flow of CNN model

\subsubsection{Convolutional layers}

These layers are responsible for extracting the visual features automatically by utilizing various kinds of filters at each layer. Nodes of every layers transmit extracted features to proceeding layers to obtain better features [39, 40].

Every layer evaluates a new feature map. The value of feature $y_{i j} \in k^{t h}$ feature map can be computed as:

$$
Z_{a b, L}=\sum\left(\boldsymbol{\omega}_{L} \odot \boldsymbol{u}_{a b}\right)+d_{L}
$$

Here, $d_{L}$ and $\boldsymbol{\omega}_{L}$ show the bias and average values of $L^{t h}$ mask. Input mask located at $(a, b)$ is represented using $\boldsymbol{u}_{a b}$. Hadamard product between matrices are represented using $\odot$. Computed coefficients are transmitted to proceeding nodes to reduce the complexity of CNN. 
Source layer

\begin{tabular}{|l|l|l|l|l|l|l|l|l|l|l|l|l|l|l|}
\hline 5 & 2 & 6 & 8 & 2 & 0 & 1 & 2 \\
\hline 4 & 3 & 4 & 5 & 1 & 9 & 6 & 3 \\
\hline 3 & 9 & 2 & 4 & 7 & 7 & 6 & 9 \\
\hline 1 & 3 & 4 & 6 & 8 & 2 & 2 & 1 \\
\hline 8 & 4 & 6 & 2 & 3 & 1 & 8 & 8 \\
\hline 5 & 8 & 9 & 0 & 1 & 0 & 2 & 3 \\
\hline 9 & 2 & 6 & 6 & 3 & 6 & 2 & 1 \\
\hline 9 & 8 & 8 & 2 & 6 & 3 & 4 & 5 \\
\hline
\end{tabular}

Figure 2: Convolutional layer process.

\subsubsection{Nonlinear layer}

This layer utilizes an activation function to handle all the nonlinear dependencies of the feature maps. An improved average biased ReLU (for details please see [41]) activation function is utilized in this paper.

\subsubsection{Pooling layer}

This layer has no direct weight, it attempts to achieve shift invariance by reducing the size of feature maps by using the activation properties of the local range of CNN. Maximum and average operations are utilized to implement the pooling layer. It considers $k \times L$ mask and computes a distinct value. For $M \times M$ layer, the output is a $\frac{M}{L} \times \frac{M}{L}$ layer.

\subsubsection{Fullyconnected layers}

Fullyconnected layers use high-level reasoning. Every input-output pair has connections with all the activations in the succeeding layer. Their activations can be evaluated with a matrix multiplication followed by a bias offset.

\subsubsection{Loss layer}

This layer is utilized to compute the trained emotion recognition model. For emotion recognition, a softmax activation function is used. Mathematiclly, loss can be computed as:

$$
k=\frac{1}{M} \sum_{m=1}^{M} \ell\left(\boldsymbol{\theta} ; \boldsymbol{z}^{(m)}, \boldsymbol{Q}^{(m)}\right)
$$


Here, $\boldsymbol{\theta}$ shows parameters of CNN model like kernel and bias operators. To compute $M$ sets, $\boldsymbol{z}^{(a)}$ is a target class composing $a^{\text {th }}$ input and $\boldsymbol{Q}^{(a)}$ represents the output of CNN.

\subsection{Nadam optimizer}

Nadam optimizer is utilized to optimize the cost function $k(\theta)$ in Algo. 1 . $\beta 1$ is utilized for decaying the mean of gradient. $\beta 2-$ is utilized for decaying the mean of the square of gradient. $\alpha \mid$ is a step size. $\in-$ is utilized to avoid division from 0 error. $f(\theta)$ is a stochastic objective function with $\theta$ parameter. $\theta_{0}$ is an initial attribute vector. $m_{0}$ and $v_{0}$ represent $1^{s t}$ and $2^{\text {nd }}$ vector, respectively. $t$ defines the timestamp. $g_{i}$ computes the gradients by considering the stochastic objective at $t . m_{i}$ and $v_{i}$ store the computed biased $1^{\text {st }}$ and $2^{\text {nd }}$ moment values, respectively. $\widehat{m_{i}}$ and $\widehat{v}_{i}$ store the evaluated bias-corrected $1^{\text {st }}$ and $2^{\text {nd }}$ moment estimates, respectively.

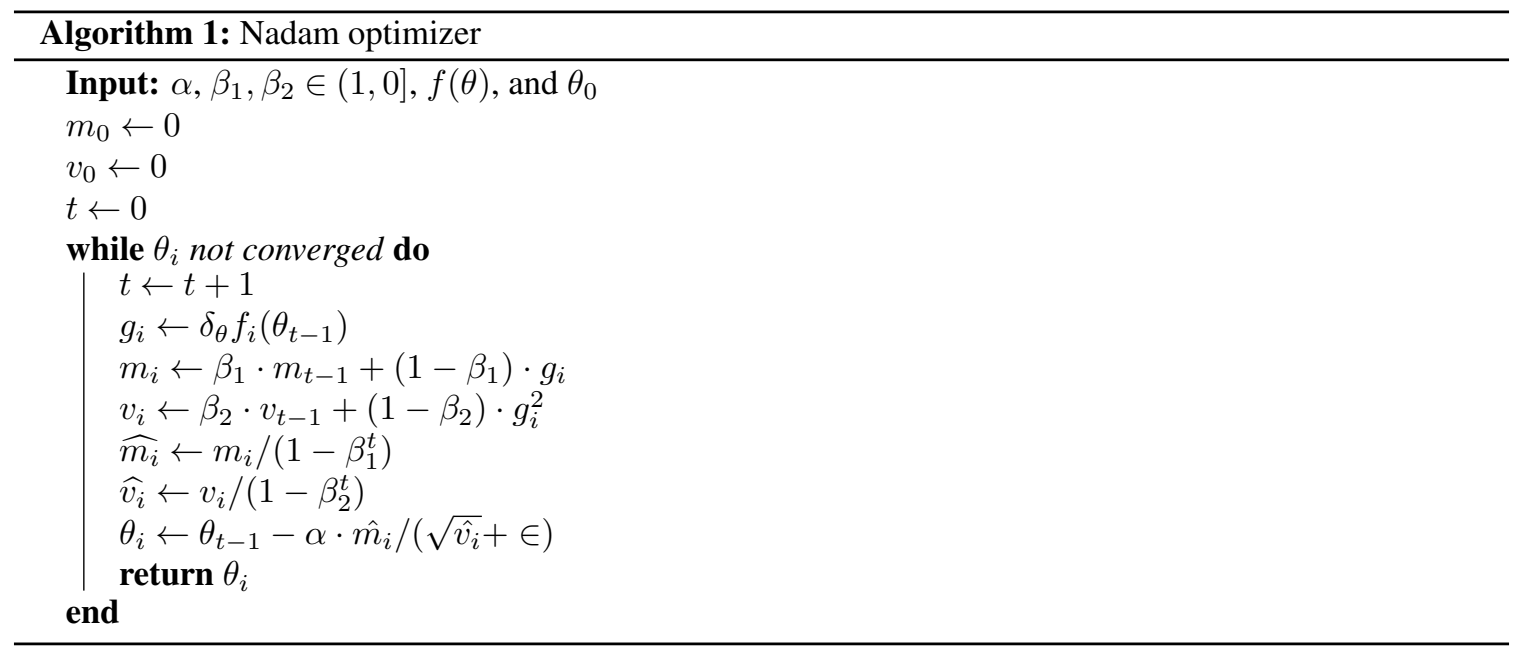

\section{Comparative analysis}

We first evaluate the proposed model on the $\mathrm{CK}+[42]$ facial emotions dataset [43], which is the most commonly used test bed for emotion recognition. Some examples of the $\mathrm{CK}+$ dataset are shown in Figure 3 Here, we can see that we have multiple numbers of emotions, but we have considered only seven features to train and test the proposed model. The selected features are as Happiness Surprise, Anger Disgust, Happiness Surprise, Fear Anger, Neutral Sadness, Fear Happiness, Anger Sadness and all other classes. These classes are labeled from 0 to 6 , respectively.

The experiments are done in MATLAB $2013 a$ on Intel core $i 7$ processor with $16-G B$ RAM. Some wellknown competitive models such as DNNs [18], CCNNRNN [19], CRAB [20], HOGLBP [21], DLP-CNN [9], and HDL [11] are considered for experimental purpose. 


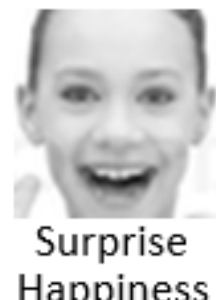

Happiness

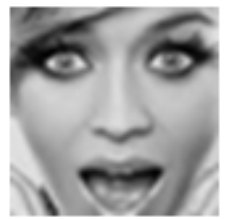

Happiness

Surprise

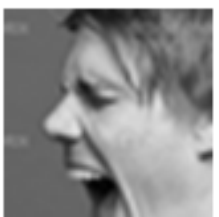

Fear

Anger

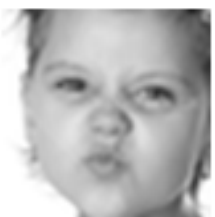

Anger

Disgust

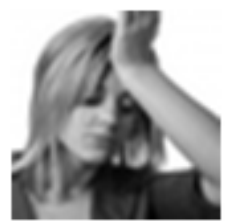

Fear

Sadness

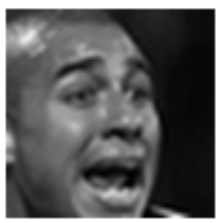

Happiness

Surprise

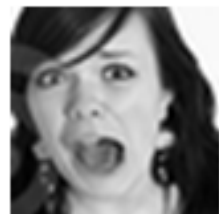

Sadness

Fear

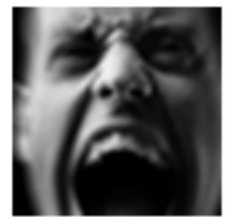

Fear

Anger

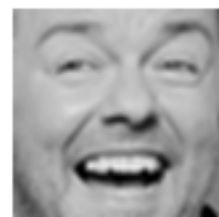

Surprise

Happiness

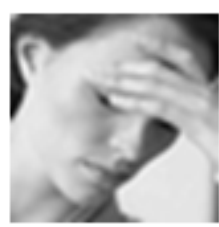

Neutral

Sadness

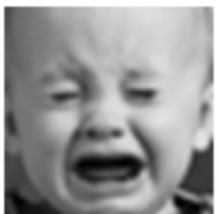

Anger

Sadness

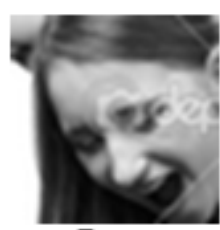

Fear

Happiness

Figure 3: Human facial emotions

\subsection{Performance metrics}

In this section, various confusion matrix-based quality metrics are discussed.

\subsubsection{Accuracy analysis}

It evaluates the ratio of the total number of correctly recognized emotions over the actual number of emotions. The accuracy $\left(A_{c}\right)$ can be defined as:

$$
A_{c}=\frac{T_{p}+T_{n}}{T_{p}+T_{n}+F_{p}+F_{n}} \times 100
$$

Here, $T_{p}, T_{n}, F_{p}$, and $F_{n}$ define true positive, true negative, false positive, and false negative values, respectively. $A_{c} \in[0,100] . A_{c}$ is desirable to be 100 .

\subsubsection{F-measure analysis}

Accuracy is not an effective measure for an imbalanced dataset. Thus, f-measure is utilized to compute the weighted mean among precision $(p)$ and recall $(r)$. Therefore, it utilizes the values of both false positives and negatives. Mathematically, F-measure can be computed as:

$$
F-\text { neasure }=2 * \frac{r * p}{r+p}
$$


where $p$ can be evaluated as:

$$
p=\frac{T_{p}}{T_{p}+F_{p}}
$$

$r$ can be computed as:

$$
r=\frac{T_{p}}{T_{p}+F_{n}}
$$

F1 Score needs to be maximized.

\subsubsection{Kappa statistic}

Cohen's kappa statistic $(\kappa)$ is a performance metric which computes the inter-rater precision (i.e., reliability) between True $(T)$ and Negative $(N)$ classes. Inter-rater precision is considered if your data raters provide a similar score to the same data item such as emotions in this research work. $\kappa$ is a more efficient measure than the simple percent agreement computation. $\kappa \in[0,1]$ and should be maximum.

\subsection{Quantitative analysis}

Figure 4 demonstrates the comparative analysis of accuracy and loss curve. The best accuracy and lesser loss is obtained during the $8^{\text {th }}$ epoch and $47^{\text {th }}$ iteration. Thus, the proposed facial emotion recognition model convergence at good speed.

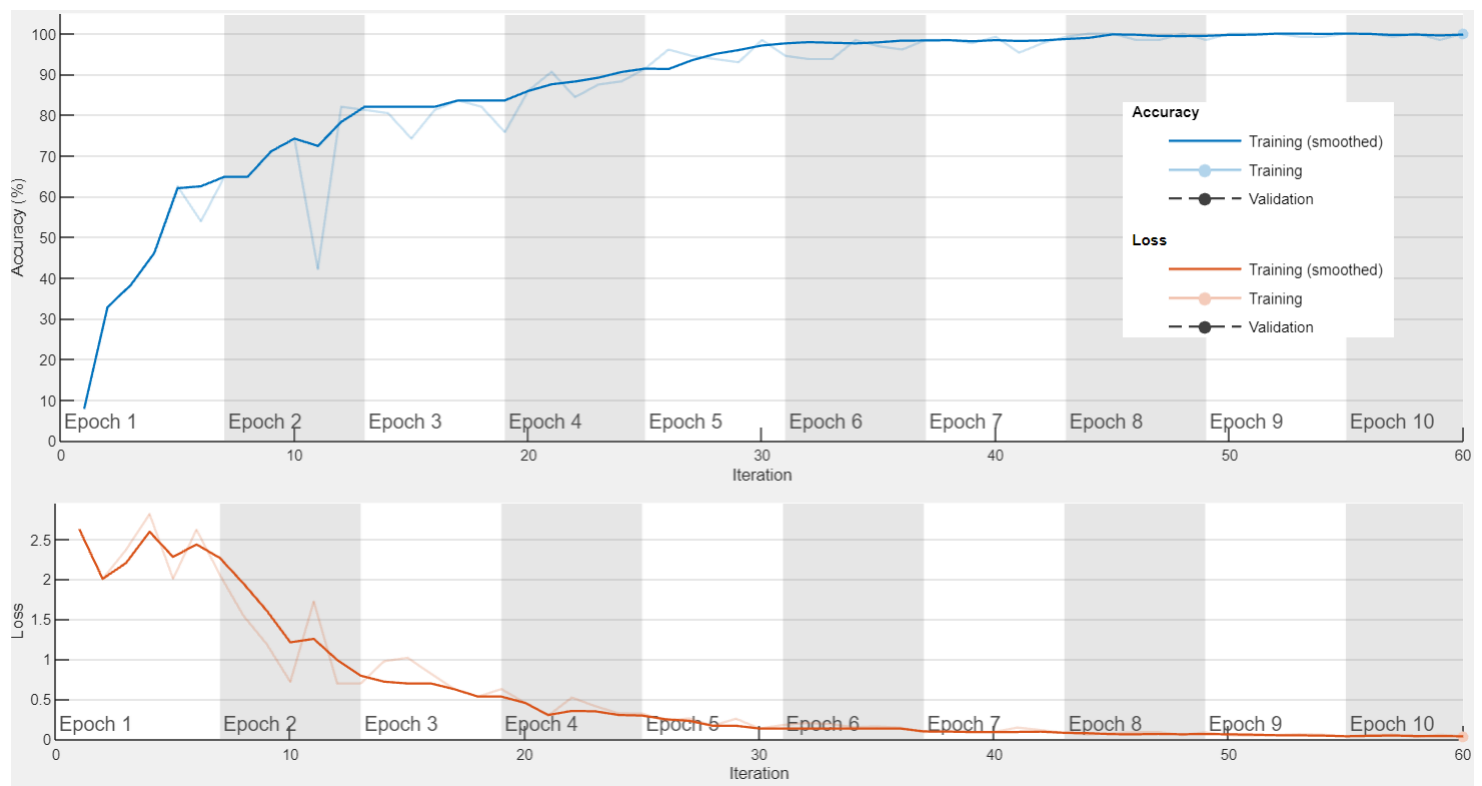

Figure 4: Comparative analysis of the proposed model

Figures 6 and 6 represent the confusion matrix of all classes of human emotions by using the proposed model with and without using CLAHE and joint trilateral filter. Since it is found that the there are majority 
of true positive and negative classes. Thus, the proposed model is least affected from the over-fitting issue.

In Figure 6, diagonal values represent the true and negative classes. Assume that class 0 is our true class, that means all other classes from 1 to 6 come under negative classes. Here, the value 27 in Figure 6 at coordinate $(0,0)$ indicates the True positive $\left(T_{p}\right)$ class, whereas the sum of all other diagonal classes shows the true negative $\left(T_{n}\right)$ class. Similarly, all vertical values represent the corresponding false values. Therefore, in this figure, when we assume the target class as anger, then we have false positives $\left(F_{p}\right)=0$ and false negatives $\left(F_{n}\right)=0$. Overall, analysis indicates that the proposed model with without joint trilateral filter and CLAHE obtains better performance as compared to without the use of joint trilateral filter and CLAHE.

\begin{tabular}{|c|c|c|c|c|c|c|c|c|}
\hline anger & $\begin{array}{c}\mathbf{2 6} \\
13.3 \%\end{array}$ & $\begin{array}{c}\mathbf{0} \\
0.0 \%\end{array}$ & $\begin{array}{c}\mathbf{0} \\
0.0 \%\end{array}$ & $\begin{array}{c}\mathbf{0} \\
0.0 \%\end{array}$ & $\begin{array}{c}\mathbf{0} \\
0.0 \%\end{array}$ & $\begin{array}{c}\mathbf{0} \\
0.0 \%\end{array}$ & $\begin{array}{c}\mathbf{0} \\
0.0 \%\end{array}$ & $\begin{array}{c}100 \% \\
0.0 \%\end{array}$ \\
\hline contempt & $\begin{array}{c}\mathbf{0} \\
0.0 \%\end{array}$ & $\begin{array}{c}11 \\
5.6 \%\end{array}$ & $\begin{array}{c}\mathbf{0} \\
0.0 \%\end{array}$ & $\begin{array}{c}\mathbf{0} \\
0.0 \%\end{array}$ & $\begin{array}{c}\mathbf{0} \\
0.0 \%\end{array}$ & $\begin{array}{c}\mathbf{1} \\
0.5 \%\end{array}$ & $\begin{array}{c}\mathbf{1} \\
0.5 \%\end{array}$ & $\begin{array}{l}84.6 \% \\
15.4 \%\end{array}$ \\
\hline disgust & $\begin{array}{c}\mathbf{0} \\
0.0 \%\end{array}$ & $\begin{array}{c}\mathbf{0} \\
0.0 \%\end{array}$ & \begin{tabular}{|c|}
$\mathbf{3 5}$ \\
$17.9 \%$
\end{tabular} & $\begin{array}{c}\mathbf{0} \\
0.0 \%\end{array}$ & $\begin{array}{c}\mathbf{0} \\
0.0 \%\end{array}$ & $\begin{array}{c}\mathbf{1} \\
0.5 \%\end{array}$ & $\begin{array}{c}\mathbf{0} \\
0.0 \%\end{array}$ & $\begin{array}{c}97.2 \% \\
2.8 \%\end{array}$ \\
\hline fear & $\begin{array}{c}\mathbf{0} \\
0.0 \%\end{array}$ & $\begin{array}{c}\mathbf{0} \\
0.0 \%\end{array}$ & $\begin{array}{c}\mathbf{0} \\
0.0 \%\end{array}$ & $\begin{array}{c}\mathbf{9} \\
4.6 \%\end{array}$ & $\begin{array}{c}\mathbf{0} \\
0.0 \%\end{array}$ & $\begin{array}{c}\mathbf{0} \\
0.0 \%\end{array}$ & $\begin{array}{c}\mathbf{0} \\
0.0 \%\end{array}$ & $\begin{array}{c}100 \% \\
0.0 \%\end{array}$ \\
\hline happy & $\begin{array}{c}\mathbf{0} \\
0.0 \%\end{array}$ & $\begin{array}{c}\mathbf{0} \\
0.0 \%\end{array}$ & $\begin{array}{c}\mathbf{0} \\
0.0 \%\end{array}$ & $\begin{array}{c}3 \\
1.5 \%\end{array}$ & $\begin{array}{c}\mathbf{4 1} \\
20.9 \%\end{array}$ & $\begin{array}{c}\mathbf{0} \\
0.0 \%\end{array}$ & $\begin{array}{c}\mathbf{0} \\
0.0 \%\end{array}$ & $\begin{array}{c}93.2 \% \\
6.8 \%\end{array}$ \\
\hline sadness & $\begin{array}{c}\mathbf{1} \\
0.5 \%\end{array}$ & $\begin{array}{c}\mathbf{0} \\
0.0 \%\end{array}$ & $\begin{array}{c}\mathbf{0} \\
0.0 \%\end{array}$ & $\begin{array}{c}\mathbf{0} \\
0.0 \%\end{array}$ & $\begin{array}{c}\mathbf{0} \\
0.0 \%\end{array}$ & $\begin{array}{c}15 \\
7.7 \%\end{array}$ & $\begin{array}{c}\mathbf{0} \\
0.0 \%\end{array}$ & $\begin{array}{c}93.8 \% \\
6.2 \%\end{array}$ \\
\hline surprise & $\begin{array}{c}\mathbf{0} \\
0.0 \%\end{array}$ & $\begin{array}{c}\mathbf{0} \\
0.0 \%\end{array}$ & $\begin{array}{c}\mathbf{0} \\
0.0 \%\end{array}$ & $\begin{array}{c}\mathbf{3} \\
1.5 \%\end{array}$ & $\begin{array}{c}\mathbf{0} \\
0.0 \%\end{array}$ & $\begin{array}{c}\mathbf{0} \\
0.0 \%\end{array}$ & $\begin{array}{c}\mathbf{4 9} \\
25.0 \%\end{array}$ & $\begin{array}{c}94.2 \% \\
5.8 \%\end{array}$ \\
\hline & $\begin{array}{c}96.3 \% \\
3.7 \%\end{array}$ & $\begin{array}{c}100 \% \\
0.0 \%\end{array}$ & $\begin{array}{c}100 \% \\
0.0 \%\end{array}$ & $\begin{array}{l}60.0 \% \\
40.0 \%\end{array}$ & $\begin{array}{c}100 \% \\
0.0 \%\end{array}$ & $\begin{array}{l}88.2 \% \\
11.8 \%\end{array}$ & $\begin{array}{c}98.0 \% \\
2.0 \%\end{array}$ & $\begin{array}{c}94.9 \% \\
5.1 \%\end{array}$ \\
\hline
\end{tabular}

Figure 5: Confusion matrix of the proposed model without joint trilateral filter and CLAHE on emotion recognition's dataset

Figures 7 to 11 demonstrate the notched boxplots analysis of the proposed model in terms of various performance metrics. The median of the evaluated parameters is demonstrated using red color. The confidence interval approaching towards median is represented using the notch of boxplot.

The accuracy analysis of the designed facial emotion recognition model is represented in Figure 7 The proposed facial emotion recognition model achieve better performance than the existing emotion recognition models. The proposed facial emotion recognition model achieves better accuracy values than the competitive models by $1.7382 \%$.

The precision analysis of the designed facial emotion recognition model is represented in Figure 8 . The 


\begin{tabular}{|c|c|c|c|c|c|c|c|c|}
\hline \multirow[b]{2}{*}{ contempt } & $\begin{array}{c}\mathbf{2 7} \\
13.8 \%\end{array}$ & $\begin{array}{c}\mathbf{0} \\
0.0 \%\end{array}$ & $\begin{array}{c}\mathbf{0} \\
0.0 \%\end{array}$ & $\begin{array}{c}\mathbf{0} \\
0.0 \%\end{array}$ & $\begin{array}{c}\mathbf{0} \\
0.0 \%\end{array}$ & $\begin{array}{c}\mathbf{0} \\
0.0 \%\end{array}$ & $\begin{array}{c}\mathbf{0} \\
0.0 \%\end{array}$ & $\begin{array}{c}100 \% \\
0.0 \%\end{array}$ \\
\hline & $\begin{array}{c}\mathbf{0} \\
0.0 \%\end{array}$ & $\begin{array}{c}11 \\
5.6 \%\end{array}$ & $\begin{array}{c}\mathbf{0} \\
0.0 \%\end{array}$ & $\begin{array}{c}\mathbf{0} \\
0.0 \%\end{array}$ & $\begin{array}{c}\mathbf{0} \\
0.0 \%\end{array}$ & $\begin{array}{c}\mathbf{2} \\
1.0 \%\end{array}$ & $\begin{array}{c}\mathbf{1} \\
0.5 \%\end{array}$ & $\begin{array}{l}78.6 \% \\
21.4 \%\end{array}$ \\
\hline disgust & $\begin{array}{c}\mathbf{0} \\
0.0 \%\end{array}$ & $\begin{array}{c}\mathbf{0} \\
0.0 \%\end{array}$ & $\begin{array}{c}\mathbf{3 5} \\
17.9 \%\end{array}$ & $\begin{array}{c}\mathbf{0} \\
0.0 \%\end{array}$ & $\begin{array}{c}\mathbf{0} \\
0.0 \%\end{array}$ & $\begin{array}{c}\mathbf{0} \\
0.0 \%\end{array}$ & $\begin{array}{c}\mathbf{0} \\
0.0 \%\end{array}$ & $\begin{array}{c}100 \% \\
0.0 \%\end{array}$ \\
\hline fear & $\begin{array}{c}\mathbf{0} \\
0.0 \%\end{array}$ & $\begin{array}{c}\mathbf{0} \\
0.0 \%\end{array}$ & $\begin{array}{c}\mathbf{0} \\
0.0 \%\end{array}$ & $\begin{array}{c}9 \\
4.6 \%\end{array}$ & $\begin{array}{c}\mathbf{0} \\
0.0 \%\end{array}$ & $\begin{array}{c}\mathbf{0} \\
0.0 \%\end{array}$ & $\begin{array}{c}\mathbf{0} \\
0.0 \%\end{array}$ & $\begin{array}{c}100 \% \\
0.0 \%\end{array}$ \\
\hline happy & $\begin{array}{c}\mathbf{0} \\
0.0 \%\end{array}$ & $\begin{array}{c}\mathbf{0} \\
0.0 \%\end{array}$ & $\begin{array}{c}\mathbf{0} \\
0.0 \%\end{array}$ & $\begin{array}{c}3 \\
1.5 \%\end{array}$ & $\begin{array}{c}\mathbf{4 1} \\
20.9 \%\end{array}$ & $\begin{array}{c}\mathbf{0} \\
0.0 \%\end{array}$ & $\begin{array}{c}\mathbf{0} \\
0.0 \%\end{array}$ & $\begin{array}{c}93.2 \% \\
6.8 \%\end{array}$ \\
\hline sadness & $\begin{array}{c}\mathbf{0} \\
0.0 \%\end{array}$ & $\begin{array}{c}\mathbf{0} \\
0.0 \%\end{array}$ & $\begin{array}{c}\mathbf{0} \\
0.0 \%\end{array}$ & $\begin{array}{c}\mathbf{0} \\
0.0 \%\end{array}$ & $\begin{array}{c}\mathbf{0} \\
0.0 \%\end{array}$ & $\begin{array}{c}15 \\
7.7 \%\end{array}$ & $\begin{array}{c}\mathbf{0} \\
0.0 \%\end{array}$ & $\begin{array}{c}100 \% \\
0.0 \%\end{array}$ \\
\hline surprise & $\begin{array}{c}\mathbf{0} \\
0.0 \%\end{array}$ & $\begin{array}{c}\mathbf{0} \\
0.0 \%\end{array}$ & $\begin{array}{c}\mathbf{0} \\
0.0 \%\end{array}$ & $\begin{array}{c}\mathbf{3} \\
1.5 \%\end{array}$ & $\begin{array}{c}\mathbf{0} \\
0.0 \%\end{array}$ & $\begin{array}{c}\mathbf{0} \\
0.0 \%\end{array}$ & $\begin{array}{c}\mathbf{4 9} \\
25.0 \%\end{array}$ & $\begin{array}{c}94.2 \% \\
5.8 \%\end{array}$ \\
\hline & $\begin{array}{c}100 \% \\
0.0 \%\end{array}$ & $\begin{array}{c}100 \% \\
0.0 \%\end{array}$ & $\begin{array}{c}100 \% \\
0.0 \%\end{array}$ & $\begin{array}{l}60.0 \% \\
40.0 \%\end{array}$ & $\begin{array}{c}100 \% \\
0.0 \%\end{array}$ & $\begin{array}{l}88.2 \% \\
11.8 \%\end{array}$ & $\begin{array}{c}98.0 \% \\
2.0 \%\end{array}$ & $\begin{array}{c}95.4 \% \\
4.6 \%\end{array}$ \\
\hline
\end{tabular}

Target Class

Figure 6: Confusion matrix of the proposed model with joint trilateral filter and CLAHE on emotion recognition dataset

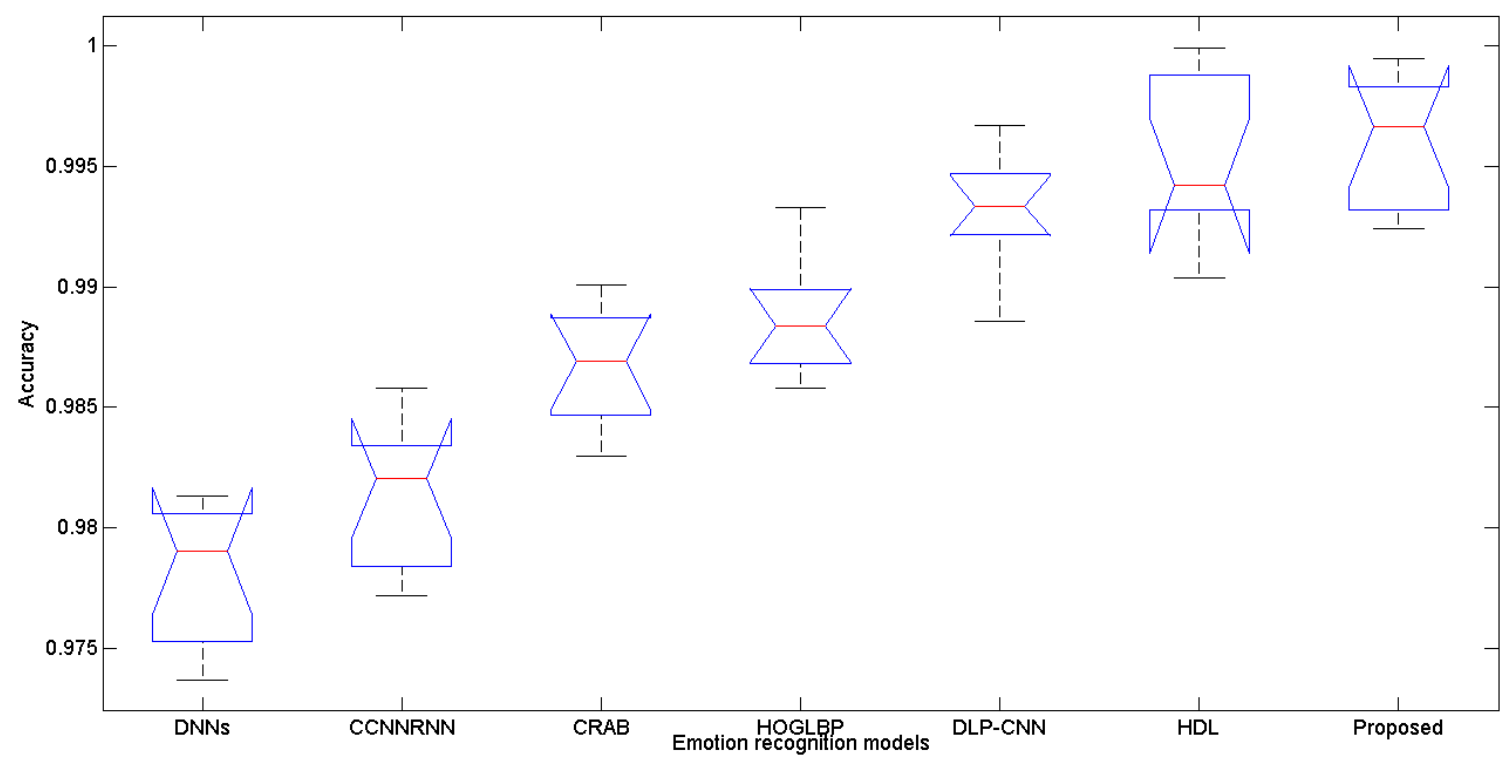

Figure 7: Accuracy analysis of the proposed facial emotion recognition model 


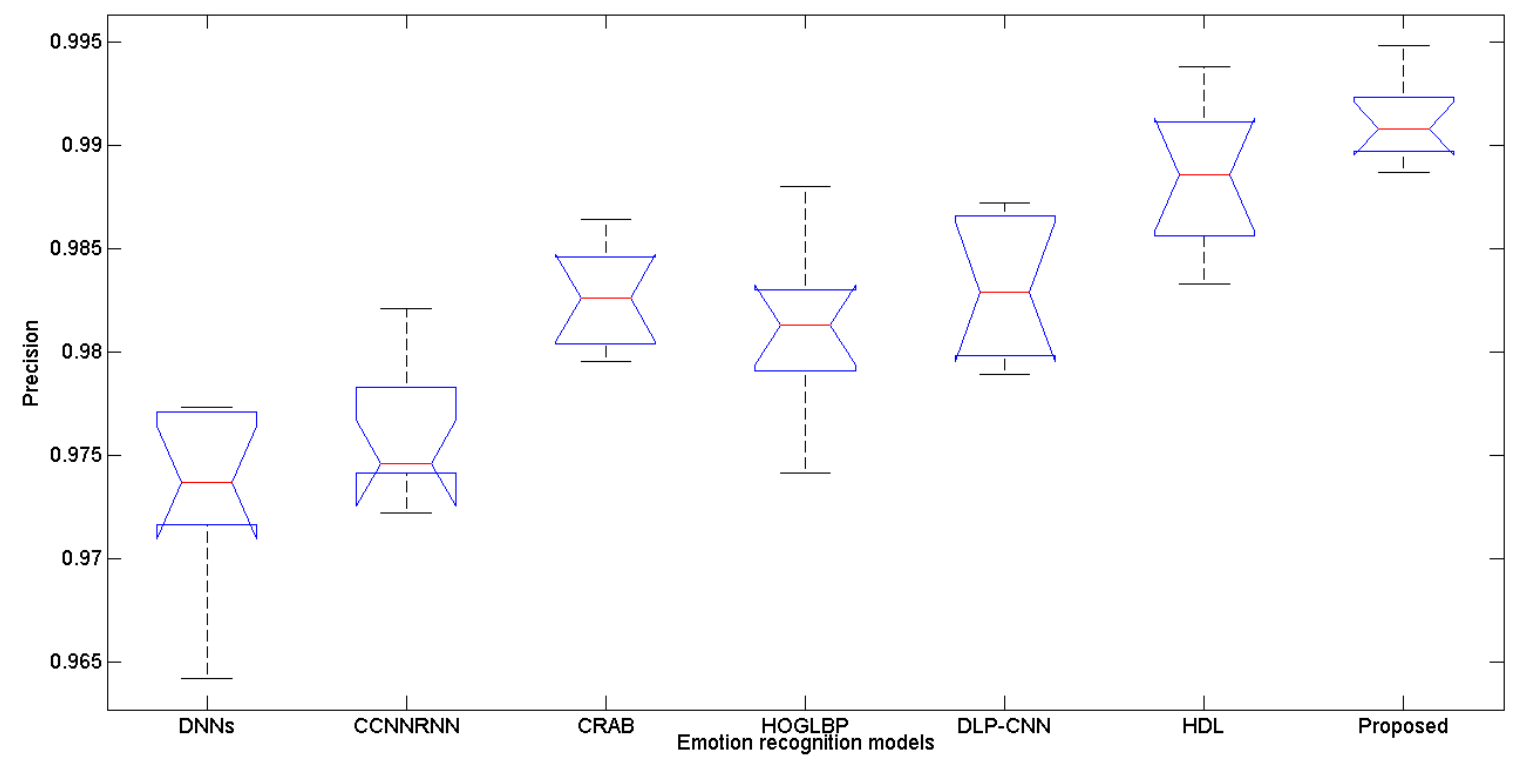

Figure 8: Precision analysis of the proposed facial emotion recognition model

proposed facial emotion recognition model achieve better precision than the existing emotion recognition models. The proposed facial emotion recognition model achieves better precision values than the competitive models by $1.3749 \%$.

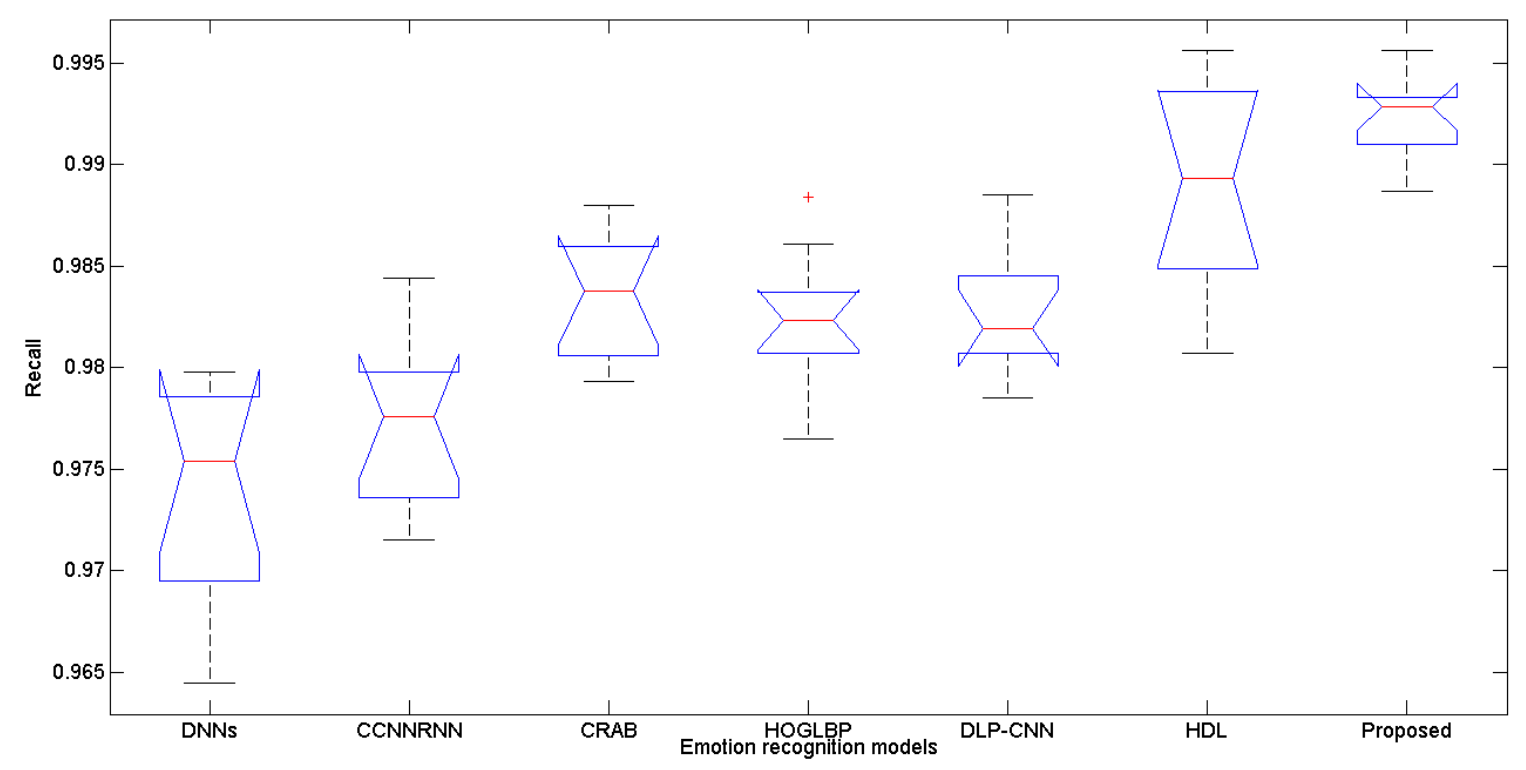

Figure 9: Recall analysis of the proposed facial emotion recognition model 
posed facial emotion recognition model achieve better recall than the existing emotion recognition models. $1.5823 \%$.

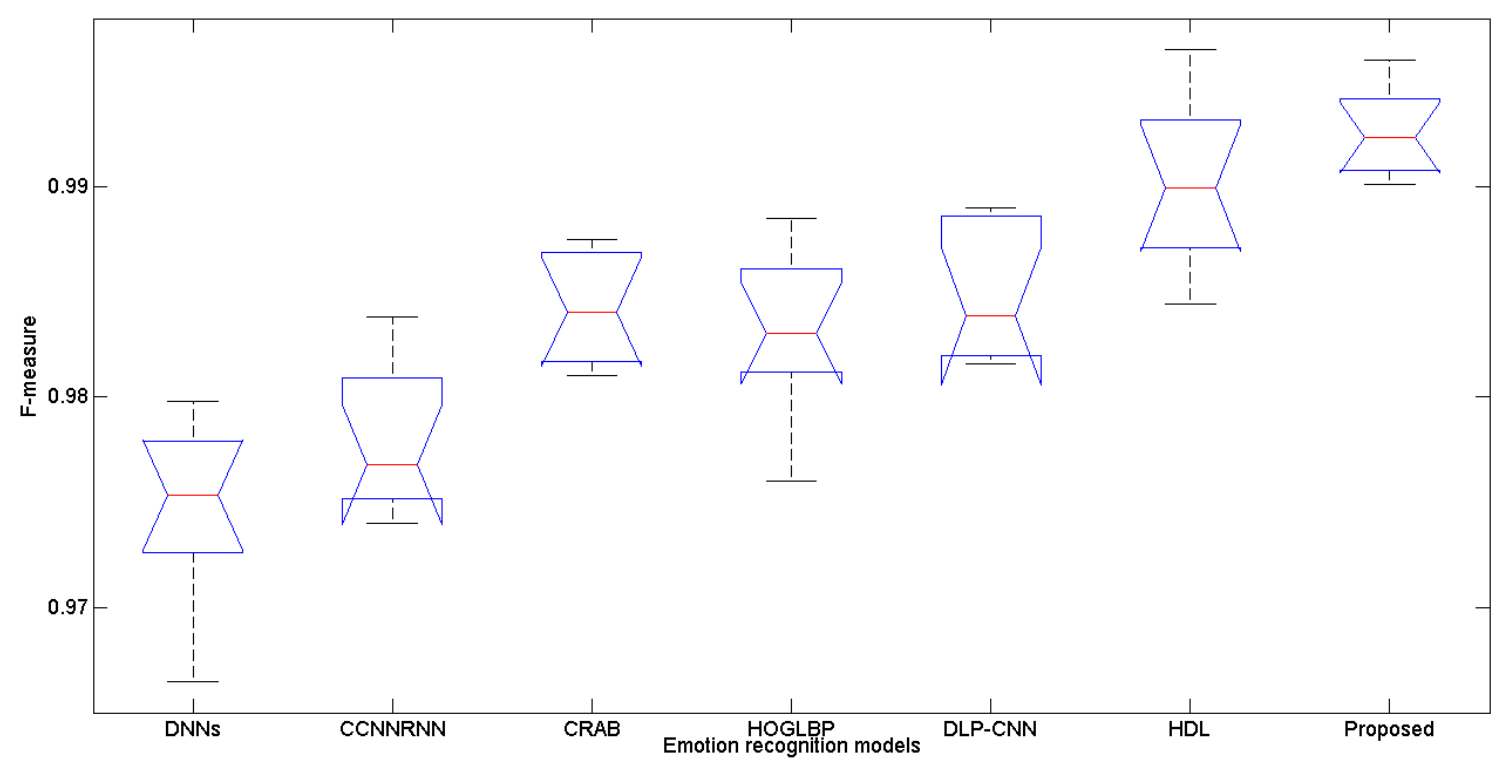

Figure 10: F-measure analysis of the proposed facial emotion recognition model

The f-measure analysis of the designed facial emotion recognition model is represented in Figure 10 The proposed facial emotion recognition model achieve better f-measure than the existing emotion recognition models. The proposed facial emotion recognition model achieves better f-measure values than the competitive models by $1.4827 \%$.

The kappa statistics analysis of the designed facial emotion recognition model is represented in Figure 11 The proposed facial emotion recognition model achieve better kappa statistics than the existing emotion recognition models. The proposed facial emotion recognition model achieves better kappa statistics values as compared to the competitive models by $1.7382 \%$.

\section{Conclusion}

It has been observed that the existing facial emotion recognition models suffer from the overfitting issue. Moreover, these models perform poorly for images which have poor visibility and noise. To handle these problems, a novel deep learning based facial emotion recognition tool was designed. Initially, a joint trilateral filter was implemented on the $\mathrm{CK}+$ facial emotions dataset to remove the noise from images. Thereafter, CLAHE was implemented on the filtered images to improve the visibility of images. Finally, the deep convolutional neural network was trained. Nadam optimizer was also used to optimize the cost function of deep 


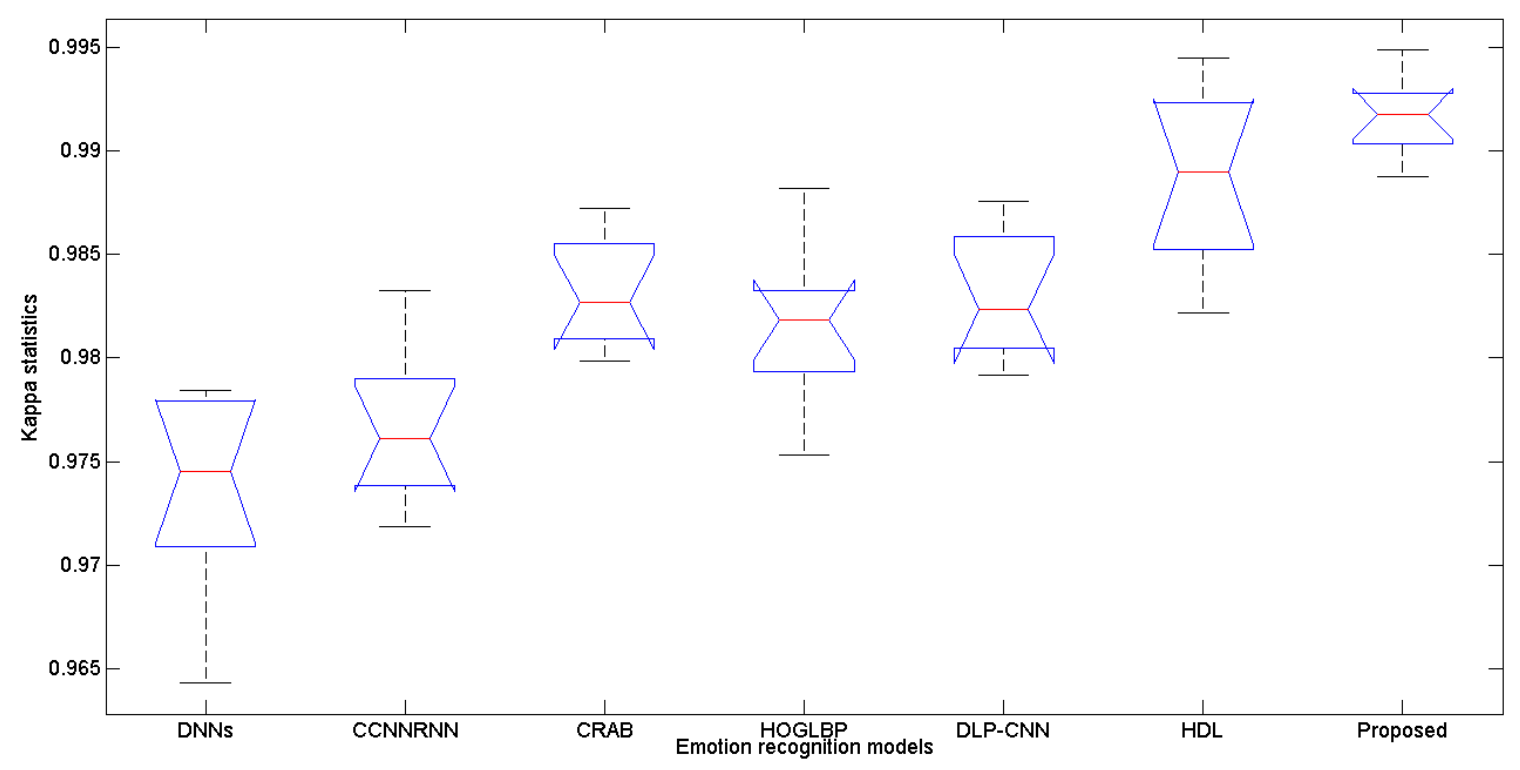

Figure 11: Kappa statistics analysis of the proposed facial emotion recognition model

convolutional neural networks. Extensive experiments were considered by using the $\mathrm{CK}+$ facial emotions dataset. The performance of the proposed technique was compared with the competitive human emotion recognition model. Comparative analysis has demonstrated that the proposed facial emotion recognition model achieves better results than the competitive models in terms of precision, accuracy, recall, f-measure, and kappa by $1.3749 \%, 1.7382 \%, 1.5823 \%, 1.4827 \%$, and $1.4563 \%$, respectively.

\section{Availability of data and materials}

The datasets generated during and/or analysed during the current study are available from the corresponding author on reasonable request.

\section{Author Contributions}

Naveen Kumari: Conceptualization, Formal analysis, Investigation, Methodology, Validation, Visualization, and Original draft preparation.

Rekha Bhatia: Project administration, Resources, Software, Supervision, Review and editing of the manuscript.

\section{Competing Interests}

The authors declare that they have no competing interests. 


\section{Funding}

None

\section{Acknowledgments}

[8] T. S. Li, P. Kuo, T. Tsai, P. Luan, Cnn and lstm based facial expression analysis model for a humanoid robot, IEEE Access 7 (2019) 93998-94011. doi:10.1109/ACCESS.2019.2928364.

[1] H. Zhang, A. Jolfaei, M. Alazab, A face emotion recognition method using convolutional neural network and image edge computing, IEEE Access 7 (2019) 159081-159089. doi:10 .1109/ACCESS . 2019.2949741.

[2] L. Gao, R. Zhang, L. Qi, E. Chen, L. Guan, The labeled multiple canonical correlation analysis for information fusion, IEEE Transactions on Multimedia 21 (2) (2019) 375-387. doi:10 .1109/TMM. 2018.2859590

[3] T. Zhang, W. Zheng, Z. Cui, Y. Zong, J. Yan, K. Yan, A deep neural network-driven feature learning method for multi-view facial expression recognition, IEEE Transactions on Multimedia 18 (12) (2016) 2528-2536. doi:10.1109/TMM.2016.2598092.

[4] W. Hua, F. Dai, L. Huang, J. Xiong, G. Gui, Hero: Human emotions recognition for realizing intelligent internet of things, IEEE Access 7 (2019) 24321-24332. doi: 10.1109/ACCESS.2019.2900231

[5] S. Zhang, X. Pan, Y. Cui, X. Zhao, L. Liu, Learning affective video features for facial expression recognition via hybrid deep learning, IEEE Access 7 (2019) 32297-32304. doi:10.1109/ACCESS. 2019.2901521.

[6] P. M. Ferreira, F. Marques, J. S. Cardoso, A. Rebelo, Physiological inspired deep neural networks for emotion recognition, IEEE Access 6 (2018) 53930-53943. doi:10.1109/ACCESS.2018. 2870063 .

[7] M. Alam, L. S. Vidyaratne, K. M. Iftekharuddin, Sparse simultaneous recurrent deep learning for robust facial expression recognition, IEEE Transactions on Neural Networks and Learning Systems 29 (10) (2018) 4905-4916. doi:10.1109/TNNLS.2017.2776248. 
[9] S. Li, W. Deng, Reliable crowdsourcing and deep locality-preserving learning for unconstrained facial expression recognition, IEEE Transactions on Image Processing 28 (1) (2019) 356-370.

[10] T. Zhang, W. Zheng, Z. Cui, Y. Zong, Y. Li, Spatial-temporal recurrent neural network for emotion recognition, IEEE Transactions on Cybernetics 49 (3) (2019) 839-847. doi:10.1109/TCYB. 2017.2788081

[11] J. Kim, B. Kim, P. P. Roy, D. Jeong, Efficient facial expression recognition algorithm based on hierarchical deep neural network structure, IEEE Access 7 (2019) 41273-41285.

[12] C.-C. Lee, E. Mower, C. Busso, S. Lee, S. Narayanan, Emotion recognition using a hierarchical binary decision tree approach, Speech Communication 53 (9-10) (2011) 1162-1171.

[13] L. Sun, S. Fu, F. Wang, Decision tree svm model with fisher feature selection for speech emotion recognition, EURASIP Journal on Audio, Speech, and Music Processing 2019 (1) (2019) 2.

[14] S. Varma, M. Shinde, S. S. Chavan, Analysis of pca and lda features for facial expression recognition using svm and hmm classifiers, in: Techno-Societal 2018, Springer, 2020, pp. 109-119.

[15] N. B. Kar, K. S. Babu, A. K. Sangaiah, S. Bakshi, Face expression recognition system based on ripplet transform type ii and least square svm, Multimedia Tools and Applications 78 (4) (2019) 4789-4812.

[16] M. Valstar, J. Gratch, B. Schuller, F. Ringeval, D. Lalanne, M. Torres Torres, S. Scherer, G. Stratou, R. Cowie, M. Pantic, Avec 2016: Depression, mood, and emotion recognition workshop and challenge, in: Proceedings of the 6th international workshop on audio/visual emotion challenge, 2016, pp. 3-10.

[17] X. Pu, K. Fan, X. Chen, L. Ji, Z. Zhou, Facial expression recognition from image sequences using twofold random forest classifier, Neurocomputing 168 (2015) 1173-1180.

[18] D. K. Jain, P. Shamsolmoali, P. Sehdev, Extended deep neural network for facial emotion recognition, Pattern Recognition Letters 120 (2019) 69-74.

[19] X. Wang, X. Chen, C. Cao, Human emotion recognition by optimally fusing facial expression and speech feature, Signal Processing: Image Communication 84 (2020) 115831. doi:https://doi.org/10.1016/j.image.2020.115831 URL https://www.sciencedirect.com/science/article/pii/ S0923596520300540

[20] A. Gupta, S. Arunachalam, R. Balakrishnan, Deep self-attention network for facial emotion recognition, Procedia Computer Science 171 (2020) 1527-1534, third International Conference on Computing and Network Communications (CoCoNet'19). 
[21] D. Lakshmi, R. Ponnusamy, Facial emotion recognition using modified hog and lbp features with deep stacked autoencoders, Microprocessors and Microsystems 82 (2021) 103834. doi:https://doi.org/10.1016/j.micpro.2021.103834, URL https://www.sciencedirect.com/science/article/pii/ S0141933121000144

22] J. C. Hung, J.-W. Chang, Multi-level transfer learning for improving the performance of 口 and named entity recognition. Applied Soft Computing 109 (2021) 107491. doi:https: //doi.org/10.1016/j.asoc.2021.107491.

u URL https://www.sciencedirect.com/science/article/pii/ S1568494621004142

[23] A. Vijaya Lakshmi, P. Mohanaiah, Woa-tlbo: Whale optimization algorithm with teaching-learningbased optimization for global optimization and facial emotion recognition. Applied Soft Computing 110 (2021) 107623. doi:https://doi.org/10.1016/j.asoc.2021.107623. URL https://www.sciencedirect.com/science/article/pii/ S1568494621005445

[24] Y. Liu, G. Fu, Emotion recognition by deeply learned multi-channel textual and eeg features, Future Generation Computer Systems 119 (2021) 1-6. doi:https://doi.org/10.1016/j. future.2021.01.010 URL https://www.sciencedirect.com/science/article/pii/ S0167739X21000200

[25] W. K. Ngai, H. Xie, D. Zou, K.-L. Chou, Emotion recognition based on convolutional neural networks and heterogeneous bio-signal data sources, Information Fusion 77 (2022) 107-117. doi:https://doi.org/10.1016/j.inffus.2021.07.007.

u URL https://wWw.sciencedirect.com/science/article/pii/ S1566253521001457

[26] Z. Zhang, C. Lai, H. Liu, Y.-F. Li, Infrared facial expression recognition via gaussian-based label distribution learning in the dark illumination environment for human emotion detection, Neurocomputing 409 (2020) 341-350. doi:https://doi.org/10.1016/j.neucom.2020.05.081. URL https://www.sciencedirect.com/science/article/pii/ S0925231220309322 
[31] G. Du, S. Long, H. Yuan, Non-contact emotion recognition combining heart rate and facial expres-

[34] W. Hua, F. Dai, L. Huang, J. Xiong, G. Gui, Hero: Human emotions recognition for realizing intelligent internet of things, IEEE Access 7 (2019) 24321-24332. doi:10.1109/ACCESS . 2019 .2900231

[35] D. Y. Choi, B. C. Song, Facial micro-expression recognition using two-dimensional landmark feature maps, IEEE Access 8 (2020) 121549-121563. doi:10.1109/ACCESS .2020.3006958. 
[36] D. Singh, V. Kumar, Dehazing of remote sensing images using improved restoration model based dark channel prior, The Imaging Science Journal 65 (5) (2017) 282-292.

[37] M. Kaur, D. Singh, V. Kumar, K. Sun, Color image dehazing using gradient channel prior and guided 10 filter, Information Sciences 521 (2020) 326-342.

[38] A. M. Reza, Realization of the contrast limited adaptive histogram equalization (clahe) for real-time image enhancement, Journal of VLSI signal processing systems for signal, image and video technology 38 (1) (2004) 35-44.

[39] N. Gianchandani, A. Jaiswal, D. Singh, V. Kumar, M. Kaur, Rapid covid-19 diagnosis using ensemble deep transfer learning models from chest radiographic images, Journal of ambient intelligence and humanized computing https://doi.org/10.1007/s12652-020-02669-6 (2020) 1-13.

[40] D. Singh, V. Kumar, V. Yadav, M. Kaur, Deep neural network-based screening model for covid-19infected patients using chest x-ray images, International Journal of Pattern Recognition and Artificial Intelligence (2020) 2151004.

[41] S. R. Dubey, S. Chakraborty, Average biased relu based cnn descriptor for improved face retrieval, Multimedia Tools and Applications (2021) 1-26.

[42] P. Lucey, J. F. Cohn, T. Kanade, J. Saragih, Z. Ambadar, I. Matthews, The extended cohn-kanade dataset (ck+): A complete dataset for action unit and emotion-specified expression, in: 2010 ieee computer society conference on computer vision and pattern recognition-workshops, IEEE, 2010, pp. 94-101.

[43] E. Barsoum, C. Zhang, C. C. Ferrer, Z. Zhang, Training deep networks for facial expression recognition with crowd-sourced label distribution, in: Proceedings of the 18th ACM International Conference on Multimodal Interaction, 2016, pp. 279-283. 